Editorial

\title{
Acknowledgment to Reviewers of Modelling in 2021
}

\author{
Modelling Editorial Office
}

Citation: Modelling Editorial Office. Acknowledgment to Reviewers of Modelling in 2021. Modelling 2022, 3, 92-93. https://doi.org/10.3390/

modelling3010006

Published: 30 January 2022

Publisher's Note: MDPI stays neutral with regard to jurisdictional claims in published maps and institutional affiliations.

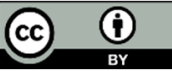

Copyright: $\odot 2022$ by the author. Licensee MDPI, Basel, Switzerland. This article is an open access article distributed under the terms and conditions of the Creative Commons Attribution (CC BY) license (https://creativecommons.org/licenses/by/4.0/).

MDPI AG, St. Alban-Anlage 66, 4052 Basel, Switzerland

Rigorous peer-reviews are the basis of high-quality academic publishing. Thanks to the great efforts of our reviewers, Modelling was able to maintain its standards for the high quality of its published papers. Thanks to the contribution of our reviewers, in 2021, the median time to first decision was 16 days and the median time to publication was 46 days. The editors would like to extend their gratitude and recognition to the following reviewers for their precious time and dedication, regardless of whether the papers they reviewed were finally published:

Adegboye, Oyelola

Ajayi, Kayode Mobolaji

Akar, Samet

Ala, Guido

AL-agele, Hadi

Almeida, Fernando

Andrejkova, Gabriela

Antosz, Katarzyna

Anvar, Amir M.

Baig, Imran

Bayati, Navid

Ben Seghier, Mohamed El Amine

Bennett, Casey

Bergant, Anton

Bernardo, Luis Filipe Almeida

Bocciarelli, Paolo

Busico, Gianluigi

Cannata, Giovanni

Caraka, Rezzy Eko

Château, Pierre-Alexandre

Chegenizadeh, Amin

Chen, Zhixiang

Cicchetti, Antonio

Cichero, Elena

Clementi, Francesco

Cochran, James J.

Correia, Anacleto

Cosma, Cosmin

Cruz, Pedro

Czarnecki, Sławomir

Delcea, Camelia

Dev, Arun

Eleftheriadis, Georgios

Elkaseer, Ahmed
El-Zohairy, Ayman

Eramo, Romina

Farahani, Behzad V.

Fedele, Rosario

Fernandes, Carlos

Ferretti, Elena

Fingas, Merv

García, Conrado Miguel Manuel

Giannopoulos, Georgios I.

Giese, Christian

Gomes, Guilherme Ferreira

Grobelna, Iwona

Hamedani, Gholamhossein

Hyunbin, Jo

Iacob, Ionut Emil

Jaliu, Codruta

Jiang, Ruinian

Jung, Robert

Kampa, Adrian

Kang, Jeon-Young

Katopodi, Irene

Klein, Louis

Kong, Byeong Yong

Lázaro, Carlos Gracia

Lesiuk, Grzegorz

Li, Gang

Li, Haipeng

Liao, Hung-Ju

Lin, Boliang

Lonetti, Paolo

Lukinac, Jasmina

Masood, Nahid Al

Mehta, Mohit

Meyer, Nils 
Milanović Glavan, Ljubica

Mosleh, Araliya

Nadhim, Evan A.

Noroozinejad, Ehsan

Ochowiak, Marek

Olaby, Osama

Pacana, Andrzej

Parsons, Jay

Perkovic, Marko

Pillai, Ajit C.

Pimenov, Danil

Połap, Dawid

Pop, Adrian

Pöschke, Florian

Quintero, Antonia M. Reina

Quirion, David

Ramos, Pedro Luiz

Reggio, Gianna

Rogulj, Katarina

Romano, Lucia

Rowlinson, Steve
Rusek, Janusz

Sana, Shib Sankar

Sánchez-Romero, Francisco-Javier

Santucci, Jean-Francois

Sarego, Giulia

Savvidis, Yiannis

Stoican, Ovidiu S.

Szeidert, Iosif

Thomas, Christian

Toba, Ange-Lionel

Toma, Ionut Ovidiu

Tomarchio, Orazio

Tsakalidis, George

Ulewicz, Robert

Vergara, Diego

Vergidis, Kostas

Wen, Chih-Yu

West, Shaun

Williams, Gustavious Paul

Zacharewicz, Gregory

Zeng, Wenhui 